\title{
The scope for monetary independence in exchange rate target zones broad and narrow
}

\author{
Margaret Hurley* \\ National University of Ireland, Maynooth, Maynooth Co. Kildare, Ireland \\ Received 15 July 1997; accepted 14 November 1997
}

\begin{abstract}
Placing limits on the movement of economic fundamentals in order to maintain an exchange rate within a target zone also puts a target zone around interest rate differentials. The bandwidth of exchange rate and interest rate differentials target zones will be different except at one point; it is suggested that this point gives a useful partitioning of target zones into broad and narrow categories. For realistic parameter values, this occurs at an exchange rate target zone of plus or minus $3.2 \%$. (C) 1998 Elsevier Science S.A.
\end{abstract}

Keywords: Exchange rates; Target zones; Bandwidths; Monetary independence; Exchange rate mechanism

JEL classification: F31; F33

\section{Introduction}

An exchange rate target zone, such as that instituted in the Exchange Rate Mechanism of the European Monetary System has the advantage of limiting exchange rate movements while still allowing for some monetary independence. However, if the width of the exchange rate target zone is widened, as happened after the European currency crisis, the scope for monetary independence ${ }^{1}$ may not increase; it depends on whether the target zone was broad or narrow to begin with. This leads to the need for a definition of what constitutes a narrow target zone and what is a broad one. As can be inferred from Svennson (1991), there is a precise relationship between the width of an exchange rate target zone and on the width of the corresponding target zone on interest rate differentials. There is a single fundamentals bandwidth that gives exchange rate zones and interest rate differentials zones of the same size; this is the suggested cutoff point between narrow and broad zones. For fundamentals target zones narrower than this, interest rate differentials have more scope for movement than exchange rates; however after this point, the position is reversed and exchange rates have more room for movement within the constraints of the policy. This cutoff point depends on the parameters of the

\footnotetext{
*Tel.: +353 1 7083787; fax: +3531 7083934; e-mail: mhurley@may.ie

${ }^{1}$ This assumes that it is valid to proxy independence by the scope for movement in interest rate differentials. This assumption affects the interpretation of results rather than the results themselves.
} 
target zone model and is calculated, using numerical methods for a number of values of these parameters.

In the original ERM the exchange rate bandwidth was plus or minus $2.25 \%$ for most countries, $6 \%$ for the others. After the currency crisis this was increased to $15 \%$. Within those official bands, there has been some discussion that countries were keeping to an unofficial narrower band of about $1 \%$ in the early 1990's, and that some are now limiting movements in the broad band to about 6\%. These exchange rate bandwidths translate to precise interest rate differential bandwidths under certain assumptions; these are the bandwidths that will be kept in mind during the discussion below.

\section{Exchange rates and interest rate differentials in target zones}

Exchange rates are assumed to be determined by the relative price of two monies, in a very simple flex-price monetary model. This model, which assumes purchasing power parity, a continuous time version of uncovered interest rate parity and stable and comparable money demand functions, says that the exchange rate is determined by fundamentals and the expected changes in exchange rates, i.e.

$$
x(t)=f(t)+\alpha E[\mathrm{~d} x(t) \mid \phi(t)]
$$

where

$x$ is the exchange rate,

$f$ is fundamentals,

$\alpha$ is the semi-elasticity of money demand,

$\phi$ is the relevant information set on which expectations are based.

The interest rate differential is, by the assumption of uncovered interest rate parity, given by

$$
\delta(t)=i(t)-i(t)^{*}=E[\mathrm{~d} x(t) \mid \phi(t)],
$$

where $i$ is the interest rate and $a^{*}$ denotes a foreign variable. The fundamental is assumed to consist of two parts; a component under the control of the government, $m(t)$, and exogenous shocks to velocity, $v(t)$.

$$
f(t)=m(t)+v(t) .
$$

Shocks hit the system via a stochastic process, that of Brownian motion with drift.

$$
\mathrm{d} v(t)=\mu \mathrm{d} t+\sigma \mathrm{d} z
$$

where $z$ is a Wiener process and $\sigma$ is a measure of the dispersion of the shocks. The exchange rate is maintained within the required target zone by changing the policy variable in response to velocity shocks; that is, limits are imposed on the movement of fundamentals. It is assumed that the policy variable is changed only at the margins and then by an infinitely small amount. As Krugman (1991) showed, the solution of the above equation, given zero drift in velocity and a symmetric target zone, is given by

$$
x(t)=f(t)-\frac{\sinh (\lambda f(t))}{\lambda \cosh (\lambda \bar{f})},
$$


where

$$
\lambda=\sqrt{\frac{2}{\alpha \sigma^{2}}} .
$$

If the bandwidth is infinite, the second term in (5) is zero; in this simple model, the free float solution is a 45 degree line. If there is a band, then taking derivatives of (5) gives Krugman's famous honeymoon effect, that is, $x_{f}<1$.

As Svennson shows, the expectation of intervention to defend the fundamentals in order to limit exchange rate movements also imposes a band on interest rate differentials. If interest rate differentials are solely determined by expectations of exchange rate changes, then they are given by

$$
\delta(f)=\frac{x(f)-f}{\alpha} .
$$

Taking derivatives of this equation with respect to fundamentals gives

$$
\delta_{f}=\frac{x_{f}-1}{\alpha}
$$

In a target zone, as shown above, the target zone exchange rate is below the $45^{\circ}$ line, and so the exchange rate slope is less than one. This implies that the interest rate differentials fundamentals relation is downward sloping and that the band on interest rate differentials is defined inversely by the band on fundamentals. With symmetry and zero fundamental drift the full solution, with the smooth pasting constants derived as described above, is

$$
\delta(f)=-\frac{\sinh (\lambda f)}{[\alpha \lambda \cosh (\lambda \bar{f})]},
$$

where the overlined $f$ is the upper intervention point on fundamentals, uniquely defined by the band on exchange rates and the policy of infinitesimal interventions. In a target zone, the interest rate differential depends both on the current level of fundamentals and on the band imposed on fundamentals by the exchange rate policy.

\section{Broad and narrow target zones}

As outlined above, defending a fundamentals bands imposes limits on both exchange rates and interest rate differentials. If the bandwidth is zero, exchange rates are fixed, and interest rate differentials are zero. As the fundamentals bandwidth increases from zero, the exchange rate and interest rate differential bandwidth also increase. In a broad target zone, for many values of fundamentals the possibility of intervention is small, and the exchange rate fundamentals relationship overlaps with the free-float 45 degree line, except at the edges of the band. The interest rate differential path will have zero slope in the middle of the band, and will be highly nonlinear at the edges of the band. From (8), if $x_{f}$ is close to one (large band), then $\delta_{f}$ is approximately zero.

In a narrow fundamentals band, the nonlinear part of the exchange rate path dominates as the probability of government intervention is always quite high (maintaining the assumption of fully 


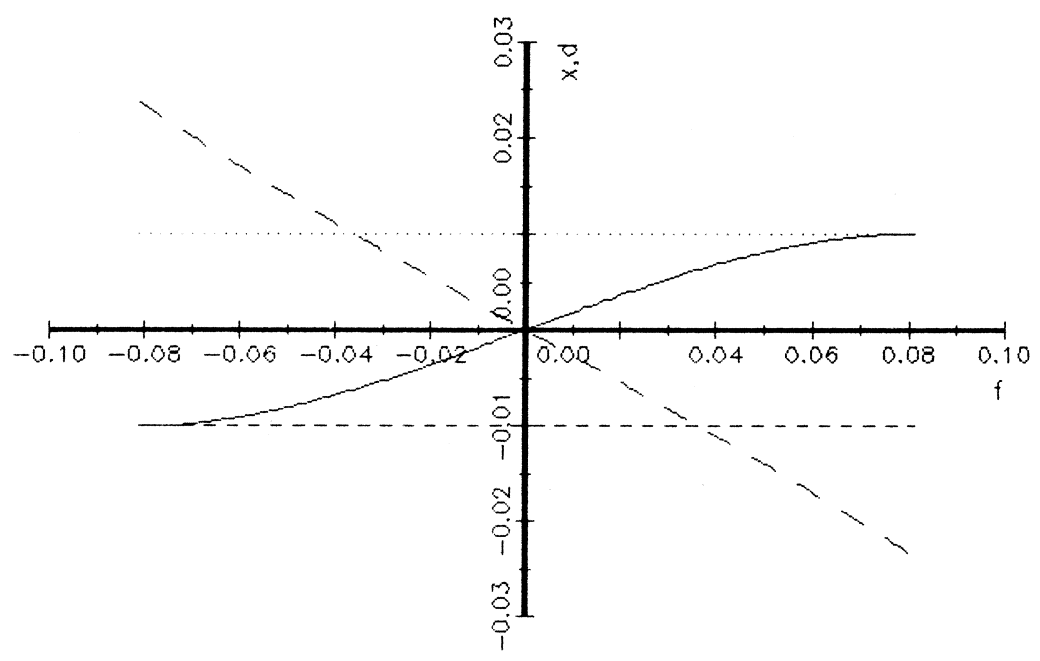

Fig. 1. $1 \%$ Exchange rate band.

credible policy). At the edges of the band, the expected change in the exchange rate following a velocity shift is zero as the government counteracts the shock. This gives an approximately linear (downward sloping) interest rate differential path, that is, $x_{f} \simeq 0$ and $\delta_{f} \simeq-1 / \alpha$. The exchange rate and interest rate differential path is drawn in Figs. 1-4 for a number of bandwidths.

If the fundamentals bandwidth is increased from zero, both the exchange rate and interest rate bandwidth obviously also increase from zero, as the variables get room to move. However, the exchange rate and interest rate bandwidth do not increase in quite the same way. From the exchange

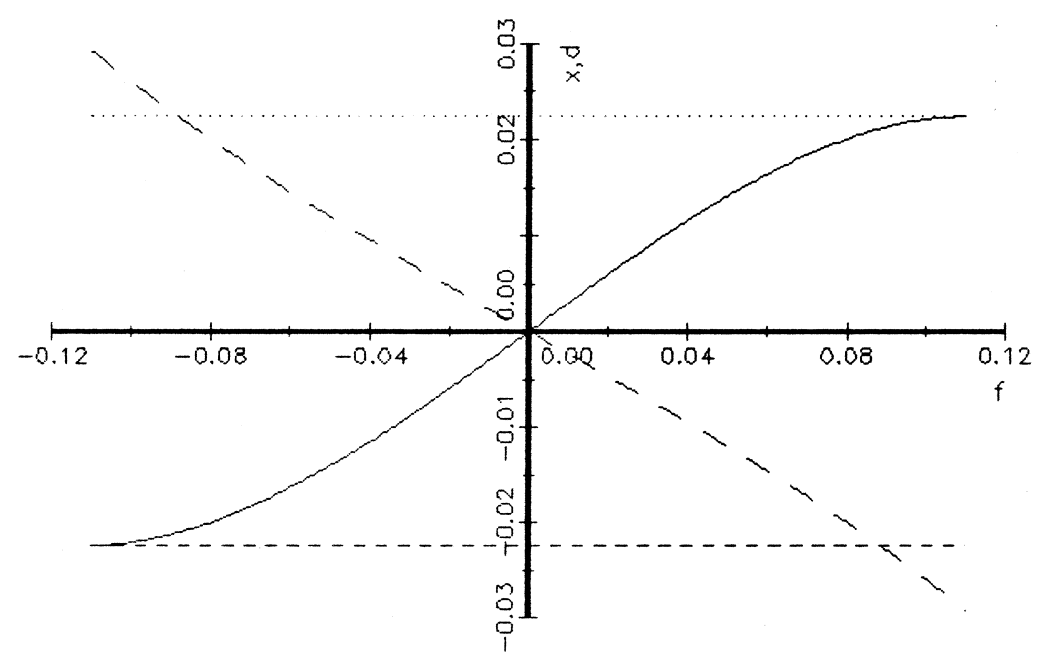

Fig. 2. 2.25\% Exchange rate band.

${ }^{2}$ These are drawn using $\alpha=3, \sigma=0.1$, values used in many of the diagrams in Svennson (1991). 


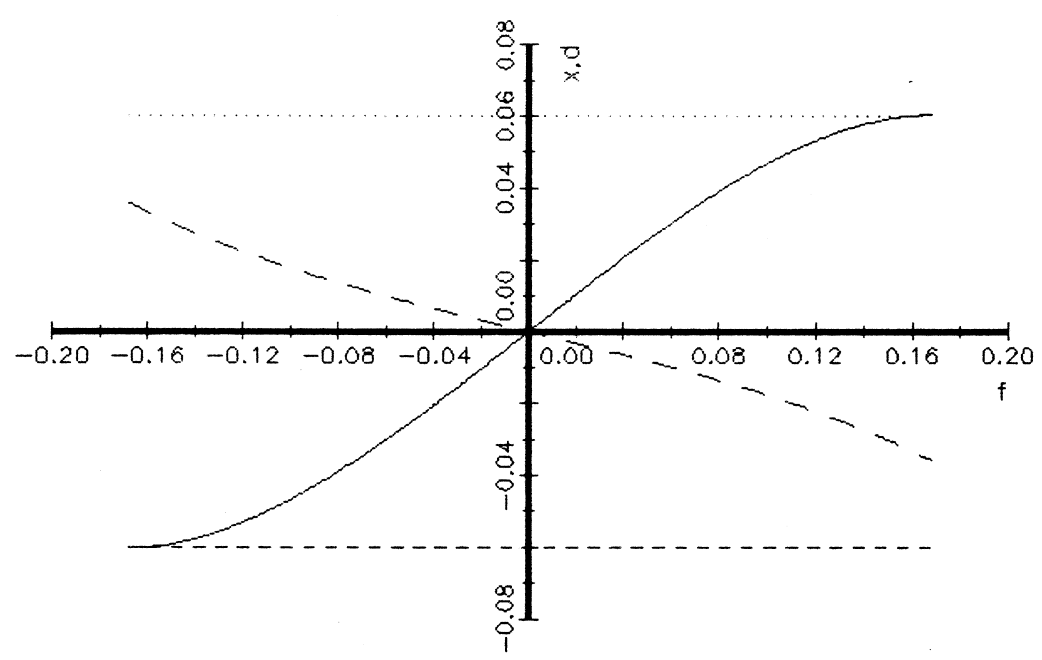

Fig. 3. $6 \%$ Exchange rate band.

rate equation above, the relationship between the exchange rate band and the fundamental band is given by

$$
\bar{x}=\bar{f}-\frac{\sinh (\lambda \bar{f})}{\lambda \cosh (\lambda \bar{f})} .
$$

The second term is positive and the hyperbolic part of it approaches one as the fundamentals band widens, implying that the exchange rate band is always narrower than the fundamentals band, and continues to widen as the fundamentals bandwidth increases.

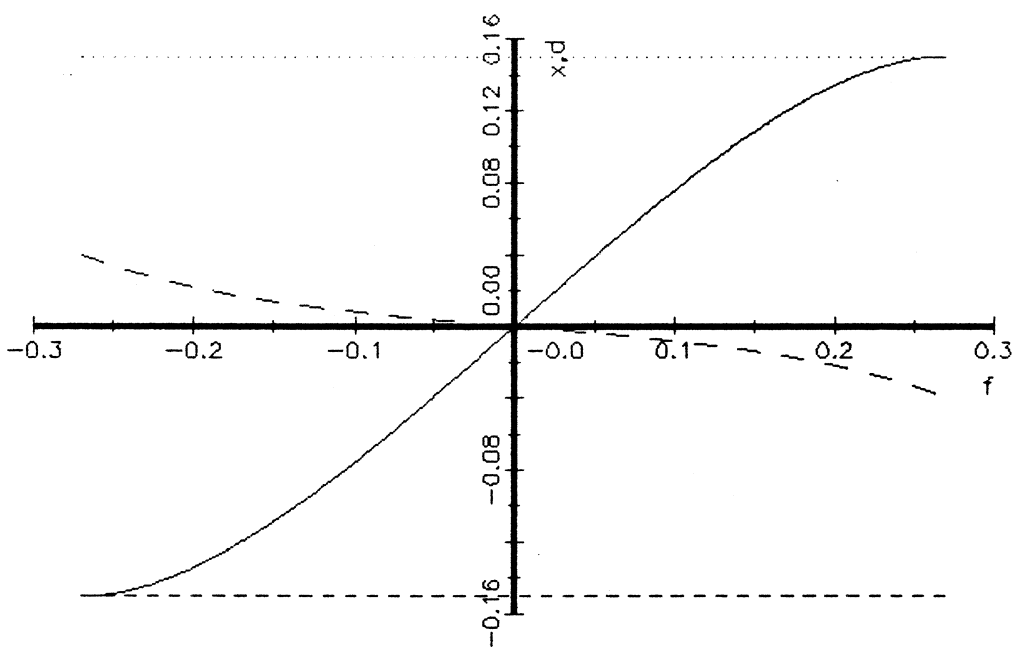

Fig. 4. $15 \%$ Exchange rate band. 


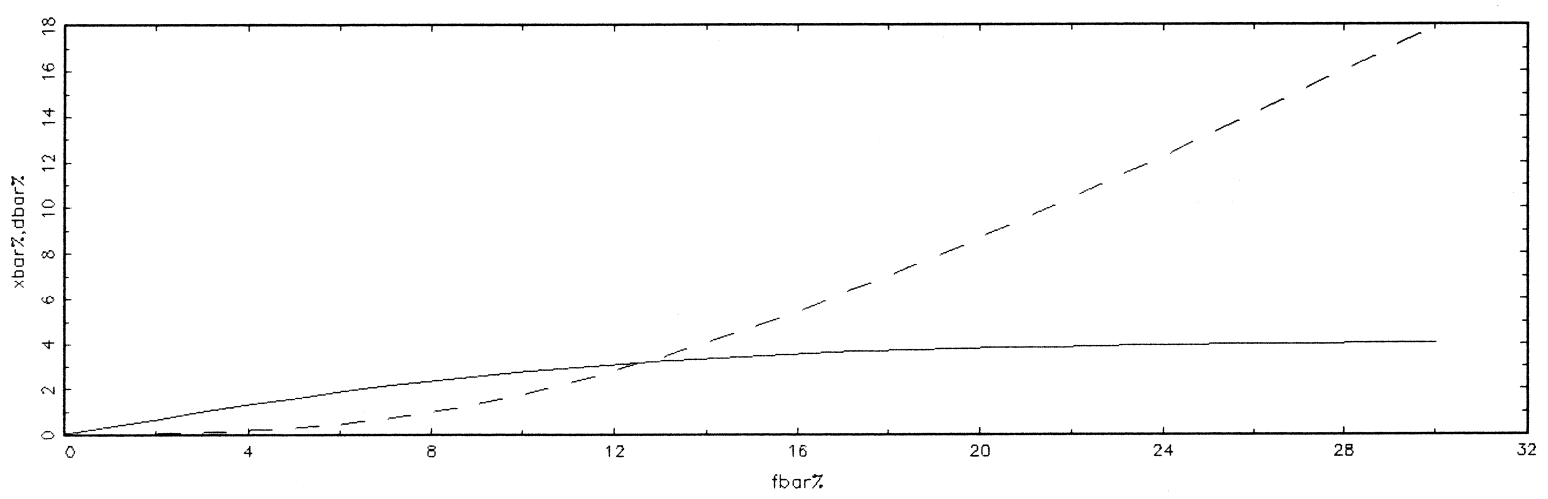

Fig. 5. Target zone bandwidths.

$$
\lim (\bar{f} \rightarrow \infty) \bar{x}=\bar{f}-1 / \lambda
$$

The interest rate differential bandwidth increases rapidly at small bandwidths, but is bounded at higher bandwidth.

$$
\begin{aligned}
& \underline{\delta}=-\frac{\sinh (\lambda \bar{f})}{\alpha \lambda \cosh (\lambda \bar{f})} \\
& \lim (\bar{f} \rightarrow \infty) \underline{\delta}=-\frac{1}{\alpha} \lambda=-\frac{\sigma}{\sqrt{2 \alpha}}
\end{aligned}
$$

As can be seen in Figs. 5 and 6, this means that changing the boundaries of a narrow target zone has quite different implications from those in a broad target zone. Changing from a completely fixed exchange rate system to a narrow target zone gives a relatively large increase in the scope of movement of interest rate differentials as opposed to the range of possible movement in exchange rates. Changing from a broad target zone to a broader target zone increases the exchange rate bandwidth proportionally with the change in the fundamentals band; the bandwidth for interest rate differentials changes little. This is a major difference in the properties of broad as opposed to narrow target zones and gives rise to my suggested categorisation of target zones by width. I define broad

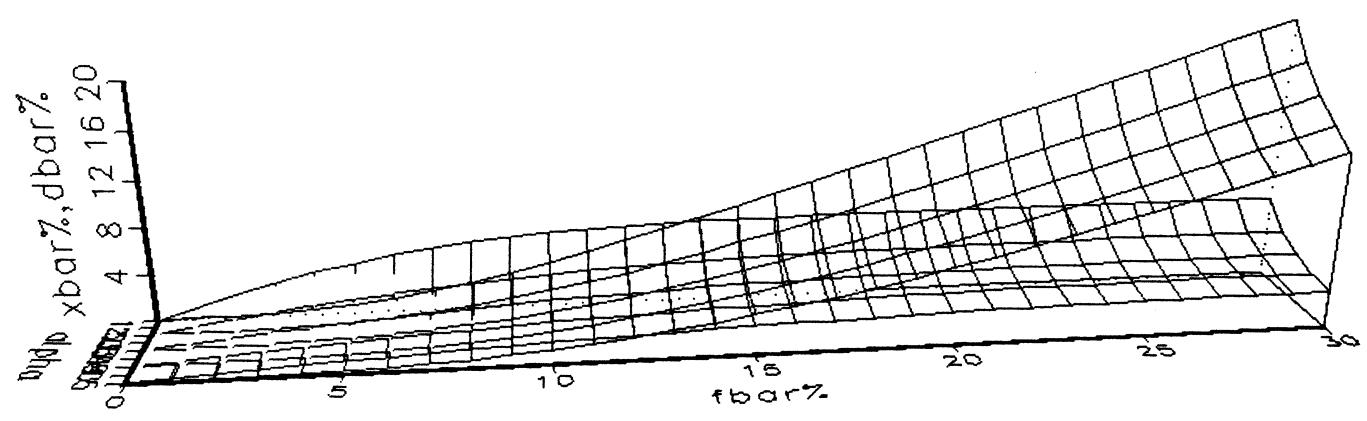

Fig. 6. Bandwidths, $a=1,2,3,4,5$. 
fundamentals target zones as those that give more scope for exchange rate changes than interest rate differential changes; narrow zones are those with a relatively large bandwidth for interest rate differentials. The cutoff point, occurs at the fundamentals bandwidth that solves the equation ${ }^{3}$

$$
\bar{f}=\frac{(1+\alpha) \sinh (\lambda \bar{f})}{\alpha \lambda \cosh (\lambda \bar{f})} .
$$

The relationship between fundamentals bandwidth and interest rate and exchange rate bandwidth depend on two parameters, $\alpha$ and $\sigma$, namely the interest rate semi-elasticity of money demand and a measure of the variability of velocity shocks. Eq. (14) is solved, using fixed point iteration, for a range of values of these parameters, and the results are reported in Table 1. Reading the highlighted line, for instance, says that for this pair of parameters values $(\alpha, \sigma)=(3,0.1)$, 'broad' target zones are those with exchange rate bandwidths of more that plus or minus $3.2 \%$, corresponding to a fundamentals bandwidth of nearly $13 \%$ either side. In this case, for example, changing the exchange rate limit from plus or minus $15 \%$ down to $6 \%$ makes very little difference to the limits of movements on interest rates, whereas changing up from a $1 \%$ to a $2.25 \%$ each side band gives a relatively large

Table 1

\begin{tabular}{|c|c|c|c|c|c|}
\hline$\alpha$ & $\sigma$ & ITER & FBAR & XBAR & DBAR \\
\hline 1 & 0.05 & 4 & 0.068 & 0.034 & 0.034 \\
\hline 1 & 0.10 & 6 & 0.135 & 0.068 & 0.068 \\
\hline 1 & 0.15 & 6 & 0.203 & 0.102 & 0.102 \\
\hline 1 & 0.20 & 5 & 0.271 & 0.135 & 0.135 \\
\hline 1 & 0.25 & 5 & 0.339 & 0.169 & 0.169 \\
\hline 2 & 0.05 & 7 & 0.064 & 0.021 & 0.010 \\
\hline 2 & 0.10 & 9 & 0.129 & 0.043 & 0.043 \\
\hline 2 & 0.15 & 9 & 0.193 & 0.064 & 0.064 \\
\hline 2 & 0.20 & 8 & 0.258 & 0.086 & 0.086 \\
\hline 2 & 0.25 & 8 & 0.322 & 0.107 & 0.107 \\
\hline 3 & 0.05 & 9 & 0.063 & 0.016 & 0.016 \\
\hline 3 & 0.10 & 12 & 0.127 & 0.032 & 0.032 \\
\hline 3 & 0.15 & 12 & 0.190 & 0.047 & 0.047 \\
\hline 3 & 0.20 & 11 & 0.253 & 0.063 & 0.063 \\
\hline 3 & 0.25 & 11 & 0.317 & 0.079 & 0.079 \\
\hline 4 & 0.05 & 11 & 0.063 & 0.013 & 0.013 \\
\hline 4 & 0.10 & 15 & 0.125 & 0.025 & 0.025 \\
\hline 4 & 0.15 & 14 & 0.188 & 0.038 & 0.038 \\
\hline 4 & 0.20 & 14 & 0.251 & 0.050 & 0.050 \\
\hline 4 & 0.25 & 14 & 0.314 & 0.063 & 0.063 \\
\hline 5 & 0.05 & 13 & 0.063 & 0.021 & 0.010 \\
\hline 5 & 0.10 & 18 & 0.125 & 0.021 & 0.021 \\
\hline 5 & 0.15 & 17 & 0.187 & 0.031 & 0.031 \\
\hline 5 & 0.20 & 16 & 0.250 & 0.042 & 0.042 \\
\hline 5 & 0.25 & 16 & 0.312 & 0.052 & 0.052 \\
\hline
\end{tabular}

${ }^{3}$ This is derived by finding the exchange rate and interest rate differential bandwidths using (10) and (12) at both upper and lower limits, and setting them equal to each other. 
increase in the scope for interest rate movements. Note also that the cutoff point increases rapidly, and linearly as the variability of velocity shocks increases. For higher values of these parameters, nearly all relevant target zones are 'narrow' by my definition.

\section{Conclusions}

This paper reviews the implications for interest rate differentials of the existence of an exchange rate target zone, keeping in mind the changes in the ERM of the EMS that have occurred during the last few years. The main point I wish to emphasise is that narrow target zones give a relatively large scope of movement of interest rate differentials; changing from a fixed to a nearly fixed exchange rate regime may give rise to large movements in interest rate differentials as a result of velocity shocks. In contrast to this, in broad band exchange rate regimes, interest rate differentials have a relatively narrow range of movement, and the interest rate differential bandwidth is not responsive to changes in exchange rate bandwidths. The distinction between broad and narrow target zones is therefore quite an important one; if monetary independence is seen as a positive outcome and exchange rate variability as a negative outcome, then narrow target zones provide relatively more of the positive and less of the negative. The results also show, that if velocity shocks are highly variable, then nearly all relevant target zones are 'narrow' by my definition. The above discussion suggests that, for less variable velocity shocks and solely from the point of view of interest rate differentials, there is very little difference between a $6 \%$ and a $15 \%$ exchange rate band.

\section{Acknowledgements}

I wish to thank Patrick Honohan and Peter Neary for helpful comments at a presentation of an earlier draft of this paper. All errors are my own.

\section{Appendix 1}

The exchange rate bandwidth is given by

$$
\bar{x}-\underline{x}=\bar{f}-f_{-}-\left[\frac{\sinh (\lambda \bar{f})}{\lambda \cosh (\lambda \bar{f})}-\frac{\sinh (\lambda \underline{-})}{\lambda \cosh (\lambda \underline{f})}\right] .
$$

Now by the assumption of symmetry

$$
f=-\bar{f}
$$

which gives

$$
\bar{x}-\underline{x}=2 \bar{f}-\left[\frac{\sinh (\lambda \bar{f})}{\lambda \cosh (\lambda \bar{f})}-\frac{\sinh (-\lambda \bar{f})}{\lambda \cosh (-\lambda \bar{f})}\right] .
$$


But since $\cosh (y)=-\cosh (y)$ and $\sinh (y)=-\sinh (-y)$ for any variable $y$, the above can be written as

$$
\bar{x}-\underline{x}=2 \bar{f}-2 \frac{\sinh (\lambda \bar{f})}{\lambda \cosh (\lambda \bar{f})} .
$$

Similarly, the interest rate bandwidth is given by

$$
\bar{\delta}-\underline{\delta}=-\frac{\sinh (\lambda \underline{f})}{\alpha \lambda \cosh (\lambda \underline{f})}+\frac{\sinh (\lambda \bar{f})}{\alpha \lambda \cosh (\lambda \bar{f})}=\frac{2 \sinh (\lambda \bar{f})}{\alpha \lambda \cosh \lambda \bar{f}}
$$

The exchange rate and interest rate differential bandwidth will be equal for the fundamentals bandwidth found by setting A3 equal to A4. This is given by

$$
\bar{f}=\frac{(1+\alpha) \sinh (\lambda \bar{f})}{\alpha \lambda \cosh (\lambda \bar{f})} .
$$

This equation says that the fundamentals bandwidth that partitions target zones into broad and narrow categories is a function of the parameters $\alpha$ and $\sigma$ (which together define $\lambda$ ). This type of equation cannot be solved to give $\mathrm{f}$ as a simple function of $\alpha$ and $\sigma$, but can be solved numerically for particular values of the parameters. This is done in a simple loop in RATS, for $\alpha=(1,2,3,4,5)$ and $\sigma=(0.05,0.1,0.15,0.2,0.25)$, values which seem to span the realistic range, to generate the table of results given in the paper.

\section{Fixed Point Iteration}

Given a function in the form

$$
x=g(x),
$$

guess an initial value called $x_{0}$. Then find

$$
x_{1}=g\left(x_{0}\right), x_{2}=g\left(x_{1}\right), \text { etc. }
$$

Stop when $x_{n}-x_{n-1}<\gamma$ where $\gamma$ is a chosen upper bound on the approximation error. Here, I initialize at $f=0.115$ and choose $\gamma=0.0001$.

\section{References}

Krugman, P., 1991. Target zones and exchange rate dynamics. Quarterly Journal of Economics 106, 669-682.

Svennson, L.E.O., 1991. Target zones and interest rate variability. Journal of International Economics 31, $27-54$. 